\title{
Treatment of Leber's hereditary optic neuropathy: An overview of recent developments
}

European Journal of Ophthalmology I-8

(C) The Author(s) 2020 Article reuse guidelines: sagepub.com/journals-permissions DOI: 10.1 | $77 / 1 \mid$ 20672/20936592 journals.sagepub.com/home/ejo (S)AGE

\author{
Marta Zuccarelli',2迆, Janis Vella-Szijj², \\ Anthony Serracino-Inglott ${ }^{1,2}$ and John-Joseph Borg ${ }^{1,3}$
}

\begin{abstract}
Leber's hereditary optic neuropathy (LHON) is a rare, maternally-inherited optic neuropathy caused by mitochondrial DNA point mutations and which can cause blindness. Currently, Raxone (idebenone) is the only available medicinal product authorised to treat LHON within the European Union and LHON remains an unmet medical need. The aim of this article was to summarise interventional clinical trials published over the past 5 years (between 2014 and 2019) with the primary purpose of treating LHON. Therapeutic approaches discussed include modulating agents of the mitochondrial electron transport chain such as Raxone, cysteamine bitartrate and KHI76, inhibitors of apoptosis such as elamipretide, gene therapy medicinal products such as GSOIO and scAAV2PIND4 and retinal tissue regeneration medicinal products such as bone marrow-derived stem cells.
\end{abstract}

\section{Keywords}

Optic neuropathy, neuro ophthalmology, genetics, hereditary/genetics factors, glaucoma, pharmacology, neuroophthalmic disease, paediatric ophthalmology

Date received: 12 December 2019; accepted: 22 April 2020

\section{Introduction}

Leber's Hereditary Optic neuropathy is a rare, maternallytransmitted optic neuropathy caused by point mutations occurring in the mitochondrial deoxyribonucleic acid (mtDNA) which affect mitochondria in retinal ganglion cells (RGCs). ${ }^{1,2}$ Point mutations m.11778G $>$ A, $\mathrm{m} .3460 \mathrm{G}>\mathrm{A}$ and $\mathrm{m} .14484 \mathrm{~T}>\mathrm{C}$ are the three most prevalent in LHON population, respectively encoding for subunits ND4, ND6 and ND1 of the mitochondrial complex I. ${ }^{3}$ Due to the mutated genes, an increased production of reactive oxygen species (ROS) occurs leading to a decreased production of ATP and causing apoptosis of RGCs. ${ }^{1}$ The point mutations m.11778G $>$ A, m.3460G $>A$ and $\mathrm{m} .14484 \mathrm{~T}>\mathrm{C}$ are also called primary mutations as they cause up to $95 \%$ of LHON cases. ${ }^{2,4}$

LHON is a rare disease with an estimated worldwide prevalence of one in 30,000 for all combined mtDNA mutations. LHON principally affects young adult males. ${ }^{4}$ Patients carrying one LHON-related mutation can manifest symptoms at any time throughout their lives, with a typical age of onset between second and third decade of life. ${ }^{4}$ Usually LHON manifests consequentially in the eyes with a rapid and painless onset of loss of central vision in one eye followed by the other eye within days to months. ${ }^{1}$ Central visual acuity (VA) declines to the level of counting fingers $(\mathrm{CF})$ in most patients. ${ }^{4}$ With disease progression, individuals usually remain legally blind for the rest of their lives with a permanent large centrocaecal scotoma. ${ }^{1}$ Cases of spontaneous recovery have been reported and found to be commonly occur with m.14484T $>\mathrm{C}$ mutation (37\% $-58 \%$ of cases) but not with mutation $\mathrm{m} .11778 \mathrm{G}>\mathrm{A}$ (4\% of cases). ${ }^{2,5}$

\footnotetext{
'Medicines Authority, San Ġwann, Malta

${ }^{2}$ Department of Pharmacy, Faculty of Medicine and Surgery, University of Malta, Msida, Malta

${ }^{3}$ Department of Biology, University of Tor Vergata, Rome, Italy

Corresponding author:

John-Joseph Borg, Medicines Authority, Sir Temi Żammit Buildings, Malta Life Sciences Park, San Ġwann, SĠN 3000, Malta.

Email: john-joseph.borg@gov.mt
} 


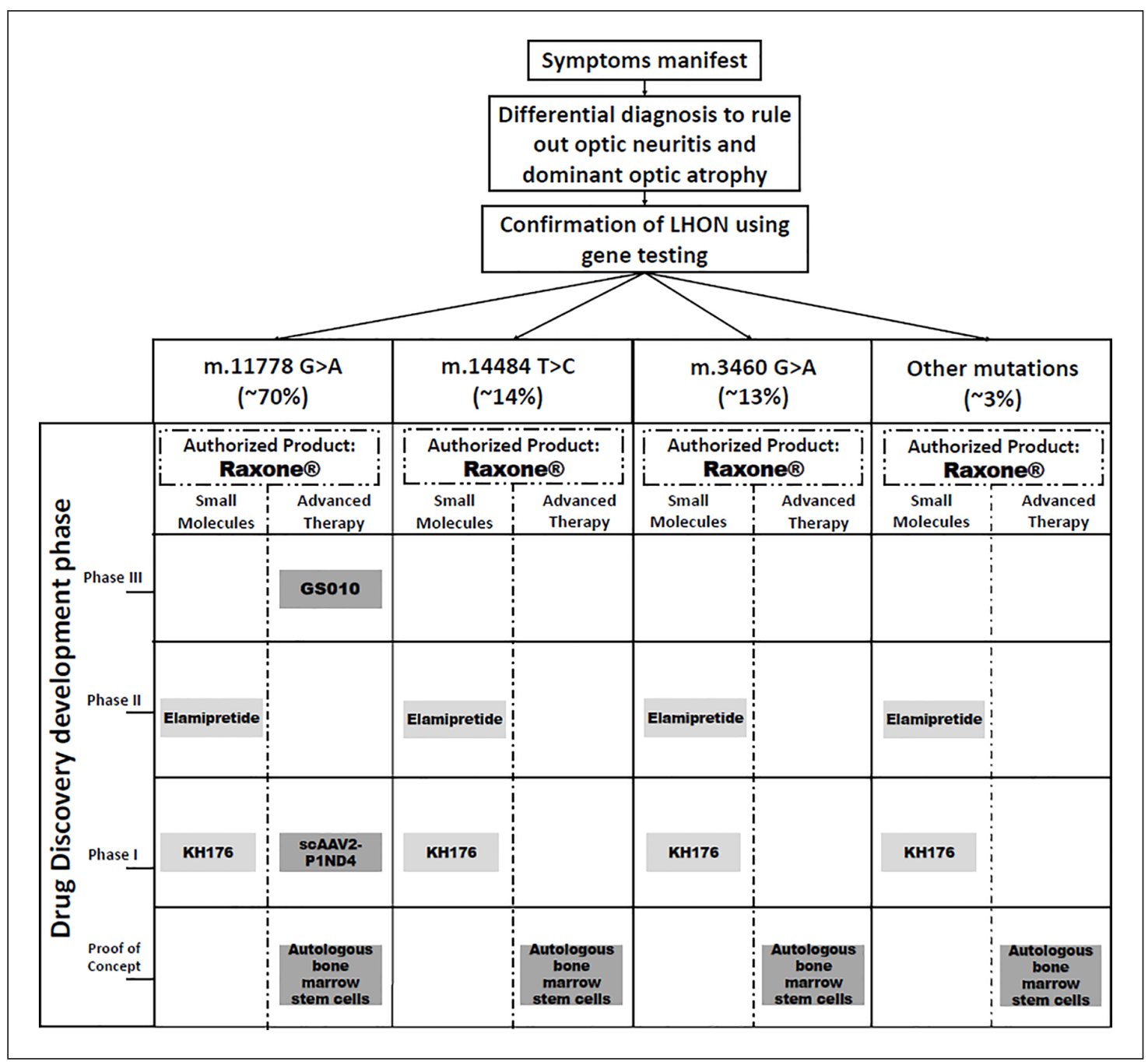

Figure I. Prospective treatment protocol for Leber's hereditary optic neuropathy $(n=5)$.

Medicinal products to treat LHON have been studied, but only few of them have been suggested to be effective. ${ }^{2,5}$ Challenges to develop medicinal products to treat LHON are present. Recruitment of naive patients in LHON clinical trials has been reported as a slow process since there are few LHON patients in the EU. Researchers have to consider an added complexity that patients carrying the m. $14484 \mathrm{~T}>\mathrm{C}$ point mutation have a $37 \%$ to $58 \%$ chance of spontaneous recovery of their VA which might introduce biases in the primary end point ${ }^{6}$ that are used to study LHON in clinical trials (best recovery in VA determined using the Early Treatment Diabetic Retinopathy Study [ETDRS] charts measured by a $\log M A R){ }^{7}$

Currently, LHON is an unmet medical need. The aim of this review was to highlight interventional clinical trials that have been published over the past 5 years, between 2014 and 2019, in the international clinical trial databases clinicaltrials.gov and clinicaltrialsregister.eu, with the primary purpose of treating LHON. A prospective treatment protocol has been proposed, including investigational medicinal products retrieved in the international clinical trials databases (Figure 1). Clinical trials have been further discussed in Table 1. Studies that have shown beneficial results in animal experiments ${ }^{8,9}$ have not been discussed.

\section{Modulating agent of mitochondrial electron transport chain}

A dysfunctional complex I (NADH-ubiquinone oxidoreductase) subunits of the mitochondrial electron transport chain (ETC) is associated with the three most common mtDNA point mutations causing LHON. ${ }^{1}$ Affecting complex I, the mutations m.11778G $>$ A, m.14484T $>$ C, $\mathrm{m} .3460 \mathrm{G}>\mathrm{A}$, lead to a deficiency of oxidative phosphorylation (OXPHOS), increased production of ROS and decreased production of ATP, eventually leading to cellular apoptosis. ${ }^{4}$ 


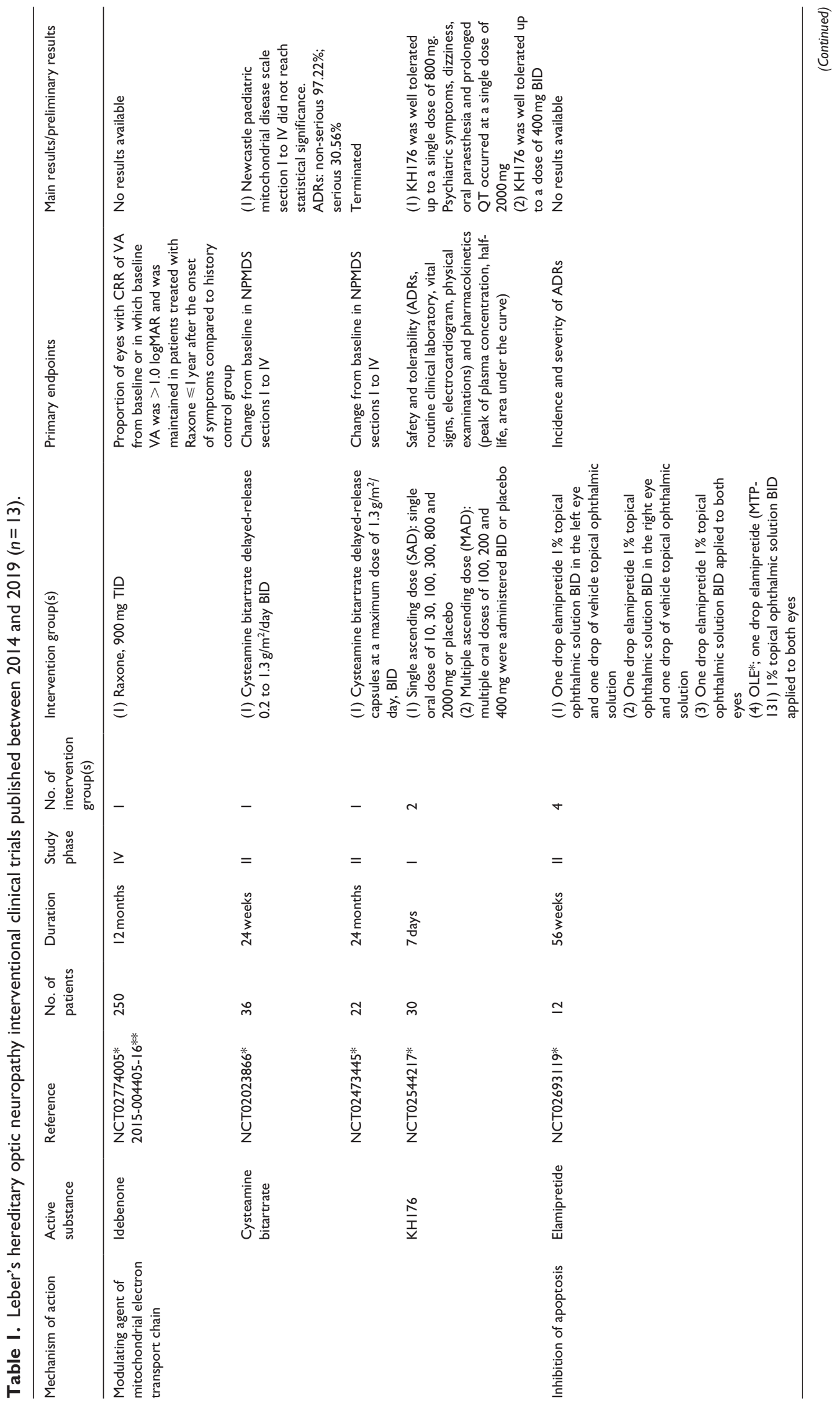




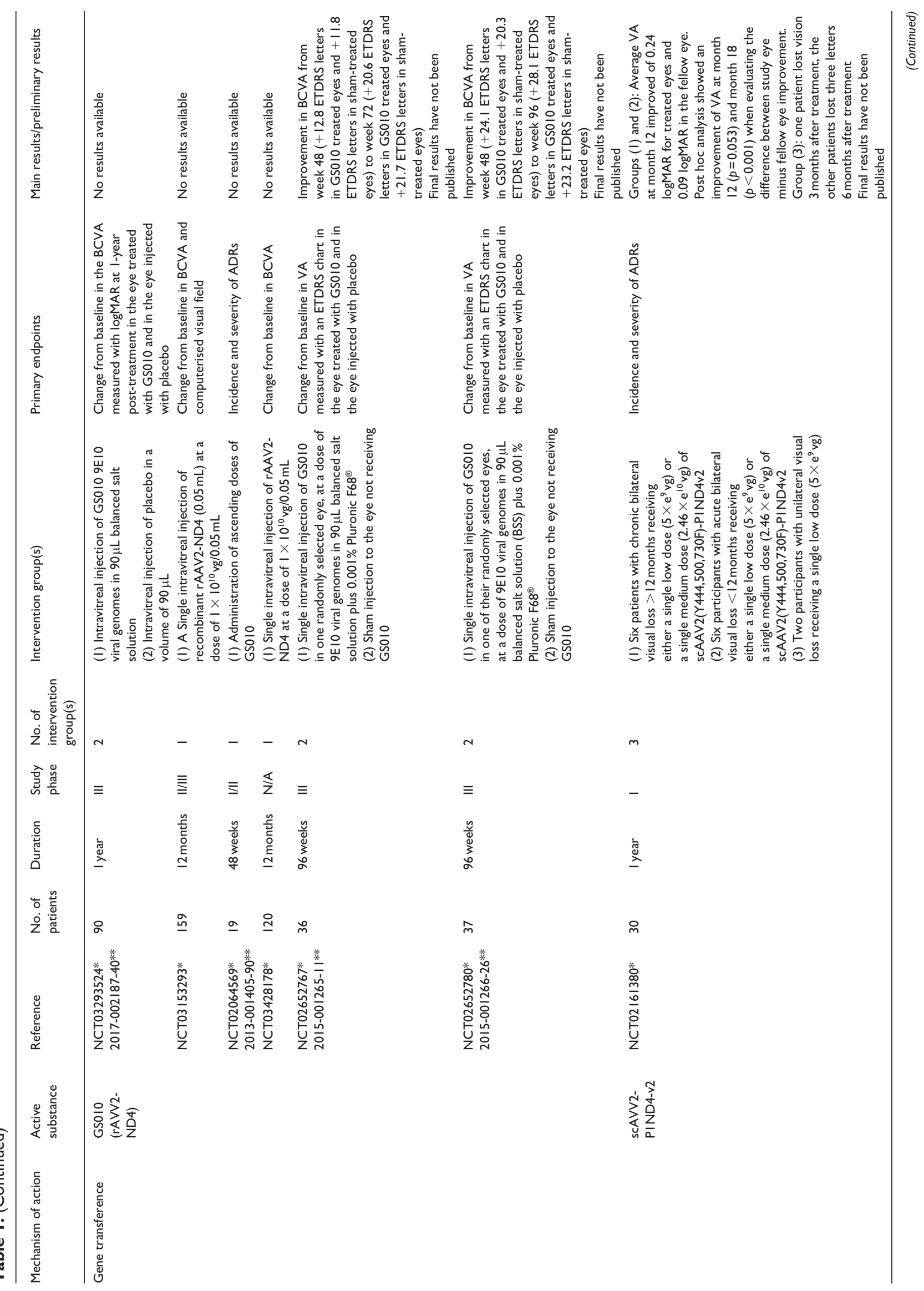




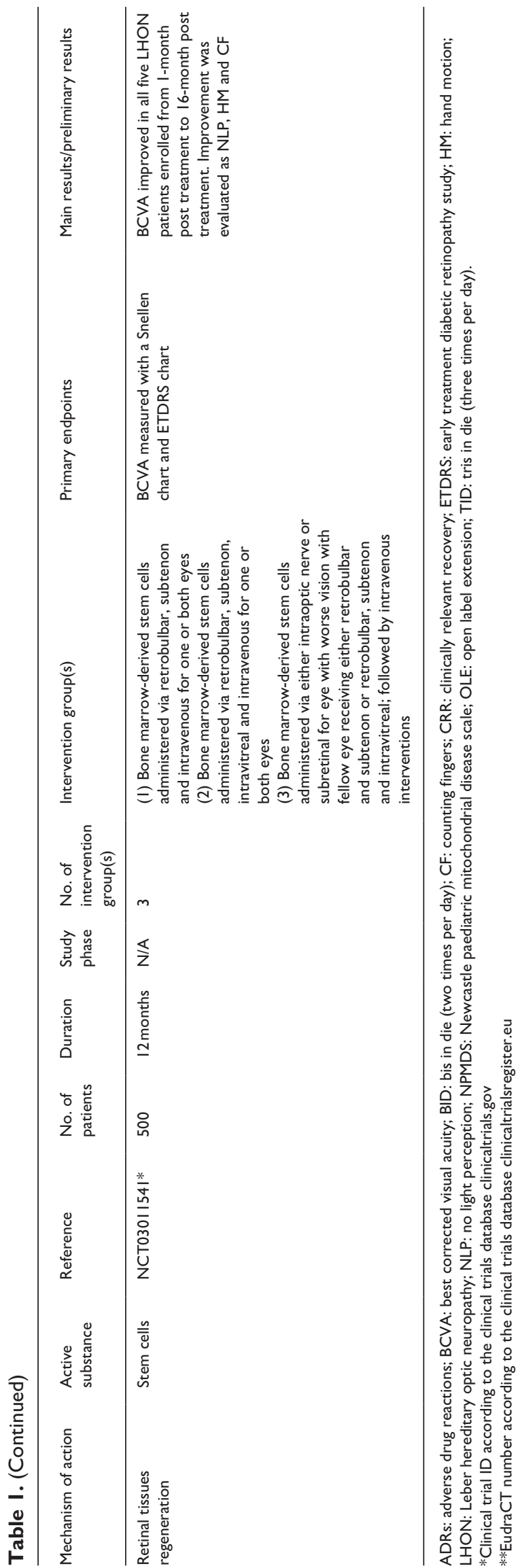

Idebenone is a short chain ubiquinone $\left(\right.$ Coenzyme $Q_{10}$ $-\mathrm{CoQ}_{10}$ ) analogue, developed in Japan for the treatment of neurodegenerative conditions. ${ }^{3}$ Idebenone is the active substance of Raxone, first medicinal product authorised to treat LHON, approved in the EU in September $2015^{3}$ (Figure 1). In the EU a marketing authorisation is required for a product to be placed on the market. ${ }^{10}$ Raxone was issued a centralised marketing authorisation due to its previous designation as orphan medicinal product. ${ }^{6}$ Raxone (idebenone $150 \mathrm{mg}$ film-coated tablets) is indicated for the treatment of visual impairment in adolescent and adult patients with LHON. ${ }^{11}$ Raxone acts as an electron carrier in the ETC, shuttling the leaking electrons onto complex III, diverting the dysfunctional complex I. In LHON, Raxone bypasses the affected complex I of the ETC, restores electron flux and ATP generation by mitigating the inactive but still viable RGCs. ${ }^{3-5}$ Raxone can promote vision recovery in LHON patients depending on time passed since manifestation of symptoms and number of RGCs already affected at the time of first administration.

The main study which led to the authorisation of Raxone was RHODOS (NCT00747487), a double-blind, randomised, placebo-controlled clinical trial. In the RHODOS clinical trial, 85 patients suffering from LHON caused by any primary mutation $(\mathrm{m} .11778 \mathrm{G}>\mathrm{A}, \mathrm{m} .3460 \mathrm{G}>\mathrm{A}$ or $14484 \mathrm{~T}>\mathrm{C}$ ) were enrolled. Patients aged between 14 and 66 and suffering from LHON for $\leqslant 5$ years were included in RHODOS and randomly assigned to receive either Raxone at a dose of $900 \mathrm{mg} /$ day or placebo for a period of 24 weeks. ${ }^{3,11}$

The main primary endpoint evaluated was the best recovery of VA (BCVA). BCVA was assessed using an ETDRS chart after 24 weeks from the eye with the best improvement in VA from baseline. 'Change in best VA' was the main secondary endpoint which evaluated the difference in VA in either the right or left eye at week 24 compared to baseline.

Results of BCVA and change in VA of the best eye were not statistically significant $(p=0.291$ and $p=0.078$, respectively). A clinically significant change in the mean VA $(p=0.026)$ was detected in a post-hoc responder analysis. Results obtained with RHODOS were further supported 4 years later, when a follow-up single-visit study (RHODOS-OFU; NCT01421381) in 58 patients showed that patients were still under the beneficial effect of Raxone also after 30 months following completion of treatment. Results of RHODOS have not been included in Table 1 because the clinical trial was carried out before 2014 . RHODOS was discussed due to the key role it played when granting a marketing authorisation for Raxone.

Further data on the use of Raxone was collected in an expanded access programme (EAP) and in a case record survey (CRS). In the EAP, 62 patients were exposed to Raxone and the number of responders to the treatment increased with longer treatment duration, $30.6 \%$ at 6 months and $36.2 \%$ at 12 months. 
Carelli et al. provided further evidence on the efficacy of Raxone in LHON patients, showing that an early start of treatment is linked to an increased visual recovery. ${ }^{7}$ Observations showed that (i) spontaneous recovery of VA is associated with a young age of symptoms onset, (ii) longer treatments were associated with vision recovery, (iii) $\mathrm{m} .11778 \mathrm{G}>\mathrm{A}$ carrier were the best responders, and (iv) involvement of the second eye was delayed in treated patients.

Further study on Raxone includes a phase IV clinical trial (post-authorisation; NCT02774005), which will evaluate the proportion of eyes with clinically relevant recovery in a 12-month period on a population of 250 patients affected from LHON caused by point mutations m.11778G $>$ A, m.3460G $>$ A and m. 14484T $>$ C. Results are not available yet (Table 1).

Other researchers have tried to enhance antioxidant medicinal products as a possible treatment for LHON. Cysteamine bitartrate has been evaluated as possible treatment for LHON due to the role that might play in reducing oxidative stress. Cysteamine bitartrate is diamine composed of a disulphide bridge, and is related to cystine, an important precursor of glutathione (GSH). Alterations in GSSG/2GSH ratio cause an increase of intracellular oxidation due to imbalance of intracellular metabolic function. ${ }^{12}$ Treatment with cysteamine bitartrate was evaluated in a clinical trial (NCT02023866) carried out on a total of 36 paediatric patients between 2 and 17 years old affected by inherited mitochondrial diseases including LHON (Table 1). Patients were administered cysteamine bitartrate delayedrelease capsules up to $1.3 \mathrm{~g} / \mathrm{m}^{2} /$ day in two divided doses, every $12 \mathrm{~h}$, for up to 6 months. The primary endpoint evaluated was change from baseline in Newcastle paediatric mitochondrial disease scale (NPMDS) sections I to IV did not reach statistical significance (section I: $p=0.1875$; section II: $p=1.0000$; section III: $p=0.0781$; section IV: $p=0.2941$ ). A high percentage of serious adverse drug reactions $(30.56 \%)$ and non-serious adverse reactions $(97.22 \%)$ occurred in the treated population. A long-term extension clinical trial was started in 2015 (NCT02473445) but was terminated due to lack of cysteamine bitartrate efficacy demonstrated in the previous study (NCT02023866; Table 1). For this reason, cysteamine bitartrate was not included in the prospective treatment protocol (Figure 1).

Another investigational medicinal product is KH176, a small molecule (a Vitamin-E-derived molecule) currently under development to treat mitochondrial-related diseases. KH176 acts as a modulating agent, targeting ROS at intracellular level. ${ }^{13} \mathrm{KH} 176$ has been studied in a phase I study (Figure 1) on healthy volunteers (NCT02544217) and the results indicate $\mathrm{KH} 176$ as safe up to a single dose of $800 \mathrm{mg}$ and multiple doses of $400 \mathrm{mg}$ twice daily but at doses of $800 \mathrm{mg}$ and $2000 \mathrm{mg}$ QT prolongations was observed $^{13}$ (Table 1).

\section{Inhibition of apoptosis of retinal ganglion cells}

In patients affected from LHON, blindness is caused by the apoptosis of RGCs. Apoptosis can be triggered by the peroxidation of cardiolipin, ${ }^{14}$ a unique anionic phospholipid which plays a key role in maintaining the curvature of mitochondria cristae. Deficiency of cardiolipin can lead to a reduced number of cristae, thus a decreased mitochondrial respiration and increased ROS production. ${ }^{9}, 15$ Under these conditions of increased ROS production, cardiolipin migrates to the outer leaflet of the inner mitochondrial membrane where undergoes peroxidation due to the activity of a cardiolipin-specific peroxidise of cardiolipinbound cytochrome $\mathrm{c}$ which promotes the release of pro-apoptotic factors. ${ }^{14,15}$

Elamipretide is a mitochondria-targeting peptide, which reduces mitochondrial ROS and cytochrome $\mathrm{C}$ release, improving mitochondrial response to metabolic changes. ${ }^{16}$ Due to its high affinity to cardiolipin, its ability to reduce oxidative stress and to prevent cytochrome peroxidase activity, elamipretide protects the architecture of mitochondria cristae. ${ }^{9}$ By protecting mitochondria, elamipretide promotes an increased ATP production and decreased ROS production.

In 2016 ReSIGHT (NCT02693119), a phase II, randomised, double-masked, vehicle-controlled clinical trial (Figure 1) was started on 12 patients suffering from LHON caused by mutation $11778 \mathrm{G}>\mathrm{A}$. The primary endpoint evaluated was the incidence and severity of adverse events occurring at 56 weeks of treatment (Table 1). Final results have not been collected yet.

\section{Gene therapy medicinal products}

About $70 \%$ of LHON patients carry the mtDNA point mutation $\mathrm{m} .11778 \mathrm{G}>\mathrm{A}$ which is associated with a low rate of spontaneous recovery ( $\sim \%$ of cases). Targetting this mutation represents the ideal target to develop medicinal products to treat $\mathrm{LHON}$.

$\mathrm{GS} 010\left(\mathrm{rAAV}_{2}-\mathrm{ND}_{4}\right)$ is a gene therapy medicinal product used to specifically treat the m.11778G $>$ A affecting the $\mathrm{ND}_{4}$ gene which is expressed only by the mitochondrial genome. GS010 delivers the missing gene using the 'allotopic expression', in which a nuclear version of the mitochondrial gene is recoded for expression and import into mitochondria using the AAV vector. ${ }^{8}$

Six clinical trials on LHON patients harbouring $\mathrm{m} .11778$ G>A have been performed between 2014 and 2019 to assess safety, efficacy and tolerability of GS010 in LHON patients. Before 2014, a clinical trial was carried out on nine LHON patients harbouring m.11778G $>$ A mutation (NCT01267422) to evaluate changes in BCVA up to 3 years post treatment (Table 1). Out of nine patients undergoing a single-dose intravitreal injection of rAAV2-ND4, six 
patients showed an improvement between 0.3 and 0.9 logMAR, two patients did not show a meaningful improvement in BCVA (improvement was $<0.3 \log \mathrm{MAR}$ ) and one patient did not show any improvement. None of the three patients who did not show improvements underwent electroretinogram examinations (one out of the three patients was too young and two out of three patients refused to have the examination), therefore the status of their retina is not known. ${ }^{2}$ The clinical trial has not been included in Table 1 because it was carried out before 2014. Clinical trials NCT01267422 has been described because of the importance it had in showing initial safety and efficacy of rAAV2-ND4.

Preliminary results of a clinical trial (clinicaltrials.gov: NCT02064569; EudraCT database 2013-001405-90) carried to evaluate safety of GS010 up to 48 weeks, showed humoral response against the viral vector (Table 1). In 2014, two randomised, parallel-assigned phase III studies, RESCUE and REVERSE (clinicaltrials.gov: NCT02652767 and NCT02652780, respectively; EudraCT database: 2015-001265-11 and 2015-001266-26, respectively) were started to evaluate the efficacy of GS010 in improving VA measured with an ETDRS chart (Table 1). In the RESCUE study, a total of 36 patients between 15 and $\geqslant 64$ years old affected from LHON caused by m.11778G $>\mathrm{A}$ and having onset of visual loss for $\leqslant 180$ days were enrolled and were randomly given an intravitreal injection into one eye of a solution containing GS010 and the other eye with a sham injection. Preliminary results at week 48 and week 72 were published from the sponsor. ${ }^{17}$ GS010-treated eyes showed an improvement from nadir at week 48 and week $72(+12.8$ letters and +20.6 letters, respectively) and from sham-injected eyes $\left(+11.8\right.$ letters and +21.7 letters, respectively). ${ }^{17}$ According to clinicaltrials.gov, database of clinical trials, final results have been collected at week 96 (September 2019). In the REVERSE study, a total of 37 patients between 15 and $\geqslant 64$ years old affected from LHON caused by m.11778G $>$ A and onset of visual loss for $>180$ days and $\leqslant 365$ days were enrolled and randomly given an intravitreal injection into one eye of a solution containing GS010 and the other eye with a sham injection. The sponsor of the study published preliminary results on REVERSE, ${ }^{18}$ showing continuous improvements in the $\log$ MAR score of patients treated eyes over 96 weeks. GS010-treated eyes showed an improvement from nadir at week 48, week 72 and week $96(+24.1$ letters, +27.4 letters and +28.1 letters, respectively) as well as sham-injected eyes $(+20.3$ letters, +22.6 letters and +23.2 letters, respectively). Final results will be collected in 2020 and data analysis will be performed.

BCVA has been further evaluated in a clinical trial (NCT03153293) carried out in 2017 in 142 LHON patients harbouring m.11778G $>\mathrm{A}$ after 12 months of GS010 treatment (Table 1). In the same year, GenSight Biologics carried out another clinical trial (NCT03293524),
REFLECT a randomised, phase III trial in which patients with vision loss onset up to 1 year were treated with bilateral intravitreal injection of GS010 (Table 1). The REFLECT study evaluates BCVA in 90 LHON patients after 12 months of GS010 treatment. In 2018, a clinical trial was started (NCT03428178) and will evaluate BCVA in patients with onset of LHON symptoms from 3 to $\geqslant 60$ months (Table 1 ).

Another approach with gene therapy is under evaluation using scAVV ${ }_{2} \mathrm{P}_{1} \mathrm{ND}_{4}$, a different gene therapy medicinal product. One non-randomised, phase I trial (NCT02161380; Figure 1) has been started in 2014 to assess toxicity of scAVV $-\mathrm{P}_{1} \mathrm{ND}_{4}$ injection on 30 patients suffering from LHON caused by mutation m.11778G $>$ A. Initial results on five patients suggested $s c A V V_{2}-P_{1} N_{4}$ to be safe. VA measured with ETDRS chart did not improve in three out of five patients, while one out of five patients had VA increase of seven letters (equivalent to three lines of vision) and one out of five patients had VA increased of 15 letters (equivalent to three lines of vision). Further results were collected on a population of 14 patients and suggested safety of $\mathrm{scAVV} \mathrm{V}_{2}-\mathrm{P}_{1} \mathrm{ND}_{4}$. Two out of 14 patients developed asymptomatic transient mild anterior uveitis which might be related to the injection of $\operatorname{sc} \mathrm{AVV}_{2}-\mathrm{P}_{1} \mathrm{ND}_{4} \cdot{ }^{19}$ VA improved between baseline and post-injection and also between day 1 post-injection and month 24 post-injection $^{19}$ (Table 1). Further data for this clinical trial will be collected.

\section{Retinal tissues regeneration}

RGCs are non-regenerative cells, therefore the loss of RGCs in LHON leads to optic nerve atrophy and patients to permanent blindness. ${ }^{5}$ No therapies are available to induce RGCs to regenerate, but researchers are evaluating possible treatments with bone marrow-derived stem cells (BMSCs), which are showing promising for neurologic and ophthalmic diseases. ${ }^{20}$

Stem Cell Ophthalmology Treatment Study (SCOTS) is an open label, non-randomised clinical trial (NCT03011541) evaluating the efficacy of BMSCs in patients suffering from ocular diseases, including LHON (Table 1). Five patients suffering from LHON caused by m.14484T $>\mathrm{C} \quad(n=2), \quad$ m.3460A $>\mathrm{G} \quad(n=2) \quad$ and m.4917A $>\mathrm{G}(n=1)$ were enrolled and BCVA using an ETDRS chart was evaluated after treatment with BMSCs. Different grade of improvement in VA and visual field had been observed in the five treated patients, occurring from 1 -month post treatment to 16 months after treatment. Preliminary results are promising, but more studies are needed to proof efficacy of BMSCs in LHON. ${ }^{20}$

\section{Conclusion}

Treatment of LHON remains a great challenge in clinical practice. Clinical trials to evaluate medicinal products to 
treat LHON are increasing in size and are being undertaken either in patients affected from mitochondrial diseases including LHON or specifically on LHON patients. According to the data obtained from the clinical trial databases clinicaltrial.gov and clinicaltrialsregister.eu, research has focused on Raxone, which is the only authorised treatment for LHON, and is now focusing on GS010, a gene therapy medicinal product which is showing promising results in treatment of LHON caused m.11778G $>$ A. Results on efficacy in long term treatment with GS010 are awaited.

\section{Authors' note}

The views expressed in this article are the personal views of the authors and may not be used or quoted as being made on behalf of, or reflecting the position of, any national competent authority, the EMA or one of its committees or working parties or any University.

\section{Declaration of conflicting interests}

The author(s) declared no potential conflicts of interest with respect to the research, authorship and/or publication of this article.

\section{Funding}

The author(s) received no financial support for the research, authorship and/or publication of this article.

\section{ORCID iD}

Marta Zuccarelli (iD) https://orcid.org/0000-0001-7577-3807

\section{References}

1. Yu-Wai-Man P, Griffiths PG and Chinnery PF. Mitochondrial optic neuropathies - disease mechanisms and therapeutic strategies. Prog Retin Eye Res 2011; 30(2): 81-114.

2. Wan X, Pei H, Zhao MJ, et al. Efficacy and safety of rAAV2ND4 treatment for Leber's hereditary optic neuropathy. Sci Rep. Epub ahead of print 19 February 2016. DOI: 10.1038/ srep21587.

3. Catarino $\mathrm{CB}$ and Klopstock $\mathrm{T}$. Use of Idebenone for the treatment of Leber's hereditary optic neuropathy: review of the evidence. J Inborn Errors Metab Screen. Epub ahead of print 18 September 2017. DOI: 10.1177/2326409817731112.

4. Yu-Wai-Man P, Soiferman D, Moore DG, et al. Evaluating the therapeutic potential of idebenone and related quinone analogues in Leber hereditary optic neuropathy. Mitochondrion 2017; 36: 36-42.

5. Jurkute $\mathrm{N}$ and Yu-Wai-Man P. Leber hereditary optic neuropathy: bridging the translational gap. Curr Opin Ophthalmol 2017; 28(5): 403-409.

6. Borg JJ, Melchiorri D, Sepodes B, et al. Optimising bench science to withstand regulatory scrutiny. Pharmacol Res 2019; 139: 491-493.
7. Carelli V, La Morgia C, Valentino ML, et al. Idebenone treatment in Leber's hereditary optic neuropathy. Brain 2011; 134(Pt 9): e188.

8. Koilkonda RD, Yu H, Chou TH, et al. Safety and effects of the vector for the Leber hereditary optic neuropathy gene therapy clinical trial. JAMA Ophthalmol 2014; 132(4): 409420.

9. Alam NM, Mills WC, Wong AA, et al. A mitochondrial therapeutic reverses visual decline in mouse models of diabetes. Dis Model Mech 2015; 8(7): 701-710.

10. Borg JJ, Laslop A, Pani L, et al. Reflections on decisions made on the well-established use of medicinal products by EU regulators and the ECJ. Sci Pharm 2014; 82(3): 541554.

11. European Medicines Agency. Raxone: EPAR - public assessment report, https://www.ema.europa.eu/en/medicines/human/EPAR/raxone (2015, accessed 22 October 2019).

12. Wilmer MJ, Kluijtmans LA, van der Velden TJ, et al. Cysteamine restores glutathione redox status in cultured cystinotic proximal tubular epithelial cells. Biochim Biophys Acta 2011; 1812(6): 643-651.

13. Koene S, Spaans E, Van Bortel L, et al. KH176 under development for rare mitochondrial disease: a first in man randomized controlled clinical trial in healthy male volunteers. Orphanet J Rare Dis 2017; 12(1): 163.

14. Manganelli V, Capozzi A, Recalchi S, et al. Altered traffic of cardiolipin during apoptosis: exposure on the cell surface as a trigger for 'antiphospholipid antibodies'. J Immunol Res 2015; 2015(7): 1-9.

15. Ren M, Phoon CK and Schlame M. Metabolism and function of mitochondrial cardiolipin. Prog Lipid Res 2014; 55: $1-16$.

16. Chatfield KC, Sparagna GC, Chau S, et al. Elamipretide improves mitochondrial function in the failing human heart. JACC Basic Transl Sci 2019; 4(2): 147-157.

17. GenSight Biologics. GenSight Biologics reports positive follow-up results at week 72 of the RESCUE phase III clinical trial of GS010 in Leber hereditary optic neuropathy (LHON), https://www.gensight-biologics.com/2019/04/17/ gensight-biologics-reports-positive-follow-up-results-atweek-72-of-the-rescue-phase-iii-clinical-trial-of-gs010-inleber-hereditary-optic-neuropathy-lhon/ (2019, accessed 22 October 2019).

18. GenSight Biologics. GenSight Biologics reports positive 96-week data from REVERSE phase III clinical trial of GS010 for the treatment of Leber hereditary optic neuropathy (LHON), https://www.gensight-biologics.com/2019/05/15/ gensight-biologics-reports-positive-96-week-data-fromreverse-phase-iii-clinical-trial-of-gs010-for-the-treatmentof-leber-hereditary-optic-neuropathy-lhon/ (2019, accessed 22 October 2019).

19. Guy J, Feuer WJ, Porciatti V, et al. Gene therapy for Leber hereditary optic neuropathy: low and medium dose visual results. Ophthalmology 2017; 124(11): 1621-1634.

20. Weiss JN, Levy S and Benes SC. Stem Cell Ophthalmology Treatment Study (SCOTS): bone marrow-derived stem cells in the treatment of Leber's hereditary optic neuropathy. Neural Regen Res 2016; 11(10): 1685-1694. 